\title{
A direct algorithm in some free boundary problems
}

\author{
Cornel Marius Murea and Dan Tiba
}

\begin{abstract}
In this paper we propose a new algorithm for the well known elliptic obstacle problem and for parabolic variational inequalities like one and two phase Stefan problem and of obstacle type. Our approach enters the category of fixed domain methods and solves just linear elliptic or parabolic equations and their discretization at each iteration. We prove stability and convergence properties. The approximating coincidence set is explicitly computed and it converges in the Hausdorff-Pompeiu sense to the searched geometry. In the numerical examples, the algorithm has a very fast convergence and the obtained solutions (including the free boundaries) are accurate.
\end{abstract}

Keywords. obstacle problem, free boundary problems, penalization.

2010 Mathematics Subject Classification. 35R35, 35J86, 35K85, 65N30, 65M12.

\section{Introduction}

Free boundary problems are described by nonlinear partial differential equations modelling important applications from physics like change of phase phenomena, contact problems in elasticity, flow propagation in porous media, etc, $[3,9]$.

Detailed theoretical discussions of various variational inequalities may be found in $[6,20,32]$. Applications, including optimal control problems are investigated in the books $[4,9,10,33]$. From the point of view of the numerical approximation, we quote just the monographs $[10,12,27]$.

Besides the solution of the given differential equation, one has to find as well the so-called free boundary, which may be a surface or a whole region (mushy region) and which is in fact the main unknown of the problem. The difficulty of such problems is related to the geometric character of this unknown and to the high nonlinearity of the corresponding equation. From this point of view, one may compare free boundary problems with shape optimization problems [26, Ch. 5.1] and, in this paper, we shall essentially use techniques from optimal design.

It is to be noticed that from the very beginning, many of the proposed solution methods for free boundary problems are of fixed domain type, in the sense that the unknown geometry is "embedded" via a certain procedure into the partial

\footnotetext{
The second author was supported by Grant 145/2011 CNCS, Romania.
} 
differential equation: the Baiocchi transform in the dam problem [3], the enthalpy method for Stefan problems [19], the regularization approach $[4,16]$ etc. The price to be paid is that the obtained partial differential equation is highly nonlinear and the free boundary has to be recovered from certain properties of the solution (for instance as a level surface). To overcome such difficulties, front tracking methods have been proposed [10,21], but their implementation may be quite complex. We also quote the discussion on error estimates and a posteriori error estimates $[1,25,27]$, the adaptive methods $[31,36]$, etc.

In this paper we propose new algorithms for the elliptic and parabolic variational inequalities which are of fixed domain type in the sense that the finite element discretization is given in the whole domain, independent of the position of the (unknown) free boundary. In each iteration a linear elliptic or parabolic equation has to be solved in the whole domain. This is a clear advantage from the point of view of the implementation and the approximating coincidence set is explicitly computed in each iteration and it converges in the Hausdorff-Pompeiu sense [26] to the searched geometry. Moreover, we need just a scalar penalization parameter in our method.

Our approach is inspired from shape optimization techniques, but no shape optimization problem is used here although this is a known method in free boundary problems, [5,23]. One may compare the present approach to the recent works $[14,24,28]$. An efficient Lagrangian method together with a primal-dual active set strategy with regularization is studied in [17] by using two perturbation parameters (except in the infeasible case) and nonlinear equations. Notice that our hypotheses in Thm. 3.3 are comparable with the conditions in [17], Thm. 3.2. See Remark 3.4 as well.

In the next section, we formulate the problems and the algorithms. Section 3 is devoted to the stability and convergence analysis. The last section reports on some numerical experiments. In the examples, the algorithm has a very fast convergence, even in the case of nonsmooth obstacles. The algorithm converges for any initial guess. The computed solutions and free boundaries are comparable with other examples of this type (via different methods) reported in the mathematical literature.

In the last example we discuss in more detail problems originating in the computation of the American options in finance (see $[2,35]$ for different approximation techniques). 


\section{Formulation of the problem and the algorithms}

\subsection{Elliptic case}

Let $D$ be a smooth domain in $\mathbb{R}^{d}, d \in \mathbb{N}^{*}=\mathbb{N} \backslash\{0\}$ and $A: H_{0}^{1}(D) \rightarrow H^{-1}(D)$ be some elliptic operator. In order to simplify the writing we shall take $A=-\Delta$ to be the Laplacian operator, but everything remains valid for general linear second order elliptic operators.

Let $\psi: D \rightarrow \mathbb{R}$ denote the obstacle function. Here, we assume $\psi \in H^{2}(D)$ and $\psi_{\mid \partial D} \leq 0$ (to be consistent with the homogeneous Dirichlet conditions). Let $f \in L^{2}(D)$ be given. The classical formulation of the obstacle problem is:

$$
\begin{aligned}
-\Delta y & =f \text { in } D^{+}=\{x \in D ; y(x)>\psi(x)\}, \\
-\Delta y & \geq f \text { in } D \backslash D^{+}=\{x \in D ; y(x)=\psi(x)\}, \\
y & =\psi \text { and } \frac{\partial y}{\partial n}=\frac{\partial \psi}{\partial n} \text { on } \partial D^{+} \cap D, \\
y & =0 \text { on } \partial D .
\end{aligned}
$$

The boundary condition (4) may be replaced by other boundary conditions. The double boundary condition (3) is due to the unknown character of the boundary $\partial D^{+}$and it determines it. The set $D^{+}$is called the noncoincidence set, while its complementary is the coincidence set. The formulation (1)-(4) corresponds to the case of strong solutions $y \in H^{2}(D) \cap H_{0}^{1}(D)$. In general, the weak formulation of (1)-(4) is given in the form of a variational inequality associated to the convex $K \subset H_{0}^{1}(D)$ :

$$
\begin{gathered}
\int_{D} \nabla y \cdot(\nabla y-\nabla v) d x \leq \int_{D} f(y-v) d x, \quad y \in K, \forall v \in K, \\
K=\left\{v \in H_{0}^{1}(D) ; v \geq \psi \text { a.e. in } D\right\} .
\end{gathered}
$$

If the elliptic operator is symmetric (which is the case for the Laplace operator), then (5)-(6) is equivalent with the variational problem

$$
\min _{v \in K}\left\{\frac{1}{2} \int_{D}|\nabla v|^{2} d x-\int_{D} f v d x\right\} .
$$

In the case where $f \in L^{2}(D), \psi \in H^{2}(D)$ with the compatibility condition $\psi_{\mid \partial D} \leq 0$, it is known that the solution of (5)-(6) satisfies the regularity property $y \in H^{2}(D)$, [4, Thm. 2.5] and the formulation (1)-(4) may be used. Moreover, in this case, the obstacle problem may be written as a multivalued equation

$$
-\Delta y+\beta(y-\psi) \ni f \text { in } D
$$


where $\beta \subset \mathbb{R} \times \mathbb{R}$ is the maximal monotone graph given by

$$
\beta(z)=\left\{\begin{array}{cc}
]-\infty, 0], & z=0 \\
0, & z>0 \\
\emptyset, & z<0
\end{array}\right.
$$

Then $\beta(y-\psi) \in L^{2}(D)$ by the maximal regularity of the solution $y \in H^{2}(D)$.

We state now our algorithm.

\section{Algorithm 1}

1) Choose $n=0, \epsilon_{0}>0, \Omega_{0} \subset D$ open, $\mathbf{y}_{-1} \in L^{2}(D)$;

2) Compute $y_{n} \in H_{0}^{1}(D)$ as solution of the linear elliptic equation

$$
-\Delta y_{n}+\frac{1}{\epsilon_{n}} \chi_{D \backslash \Omega_{n}}\left(y_{n}-\psi\right)=f \text { in } D
$$

(here $\chi_{D \backslash \Omega_{n}}$ is the characteristic function of $D \backslash \Omega_{n}$ where it takes the value 1 and 0 otherwise, corresponding to the approximation of the coincidence set in iteration $n)$;

3) $\mathbf{y}_{n}=\max \left\{y_{n}, \psi\right\}, \Omega_{n+1}=\left\{x \in D ; \mathbf{y}_{n}(x)>\psi(x)\right\}, \epsilon_{n+1}=\frac{\epsilon_{n}}{2}$;

4) If $\left\|\mathbf{y}_{n}-\mathbf{y}_{n-1}\right\|_{L^{2}(D)}<$ tol then STOP else $\mathrm{n}=\mathrm{n}+1$ GO TO step 2.

Remark 2.1. Notice that by the regularity theory for elliptic equations, in Step 2 we have $y_{n} \in H^{2}(D) \cap H_{0}^{1}(D)$. Then, by the Sobolev theorem in dimension $d \leq 3$, we get $y_{n} \in \mathcal{C}(\bar{D})$ and $\Omega_{n}$ defined in Step 3 is open, for any $n \in \mathbb{N}$.

Remark 2.2. The algorithm uses just linear elliptic equations in the whole domain $D$. The type of penalization term from Step 2 may be compared with the approach developed in shape optimization problems in [24]. A classical nonlinear penalization term for solving the obstacle problem is $\frac{1}{\epsilon}\left(y_{n}-\psi\right)^{-}$where $v^{-}(x)=-v(x)$ if $v(x)<0$ and $v^{-}(x)=0$ if $v(x) \geq 0$, see for example [12]. Another advantage of the Algorithm 1 is that it approximates the coincidence set explicitly. We also underline its simplicity.

Remark 2.3. By the classical result of [7], the elastic-plastic torsion problem is equivalent with a variational inequality of obstacle type and our algorithm may be applied as well. In [13], a related problem is studied by a comparable approach involving semi-smooth Newton methods. Similar results may be obtained for the bilateral obstacle.

Remark 2.4. In the numerical experiments, we also use the energy norm in the stopping test or a fixed small parameter $\epsilon_{n}$ in all the iterations. 
Let $\beta_{\epsilon}: \mathbb{R} \rightarrow \mathbb{R}$ denote the Yosida approximation of $\beta$ given by (8) that is:

$$
\beta_{\epsilon}(r)=\left\{\begin{array}{cc}
r / \epsilon, & r \leq 0 \\
0, & r>0
\end{array}\right.
$$

Extending its derivative by $1 / \epsilon$ in $x=0$, we can rewrite the step 2 of the Algorithm 1 as

$$
-\Delta y_{n}+\left(\beta_{\epsilon_{n}}^{\prime}\left(y_{n-1}-\psi\right)\right)\left(y_{n}-\psi\right)=f .
$$

In (10), we take into account step 3 as well. Recall that the usual approximation by regularization of the variational inequality (5)-(6) is

$$
-\Delta \tilde{y}_{n}+\beta_{\epsilon_{n}}\left(\tilde{y}_{n}-\psi\right)=f \text { in } D
$$

plus homogeneous boundary conditions on $\partial D$. Notice that $\beta_{\epsilon}(r)=\beta_{\epsilon}^{\prime}(r) r$, under the above extension convention, which shows that (10) and (11) have very similar structure. Clearly, (11) is a nonlinear elliptic equation, while the decoupling operated in (10) allows to use linear elliptic equations. The form (9) puts into evidence the approximating coincidence set too.

Moreover, we have $y_{n}(x) \leq \tilde{y}_{n}(x)$ a.e. in $D$. To infer this, we use $\beta_{\epsilon}(r)=$ $\beta_{\epsilon}^{\prime}(r) r$, the concavity of $\beta_{\epsilon}(\cdot)$ and the definition of the subdifferential of concave mapping:

$$
\begin{gathered}
\left(\beta_{\epsilon_{n}}^{\prime}\left(y_{n-1}-\psi\right)\right)\left(y_{n}-\psi\right)=\left(\beta_{\epsilon_{n}}^{\prime}\left(y_{n-1}-\psi\right)\right)\left(y_{n-1}-\psi\right) \\
+\left(\beta_{\epsilon_{n}}^{\prime}\left(y_{n-1}-\psi\right)\right)\left(y_{n}-\psi-y_{n-1}+\psi\right) \geq \beta_{\epsilon_{n}}\left(y_{n-1}-\psi\right) \\
+\beta_{\epsilon_{n}}\left(y_{n}-\psi\right)-\beta_{\epsilon_{n}}\left(y_{n-1}-\psi\right)=\beta_{\epsilon_{n}}\left(y_{n}-\psi\right) .
\end{gathered}
$$

We get

$$
-\Delta y_{n}+\beta_{\epsilon_{n}}\left(y_{n}-\psi\right) \leq f
$$

by the monotonicity of $\beta_{\epsilon}(\cdot)$. This ends the argument by subtracting (11) from (12) and multiplying by $\left(y_{n}-\tilde{y}_{n}\right)_{+}$.

\subsection{Parabolic case}

Let $T>0$ be given.

Let $\psi \in L^{2}\left(0, T ; H^{2}(D)\right) \cap H^{1}\left(0, T ; L^{2}(D)\right)$ be given such that $\psi(t, x) \leq 0$ a.e. on $[0, T] \times \partial D$. We associate with it the closed convex sets

$$
\begin{aligned}
\mathcal{K}(t) & =\left\{v \in H_{0}^{1}(D) ; v(x) \geq \psi(t, x) \text { a.e. } D\right\} \\
\mathcal{K} & =\left\{v \in L^{2}\left(0, T ; H_{0}^{1}(D)\right) ; v(t, x) \geq \psi(t, x) \text { a.e. }[0, T] \times D\right\} .
\end{aligned}
$$


The following variational inequalities (that may be termed as parabolic obstacle problems) will be considered for $f \in L^{2}([0, T] \times D)$ :

$$
\begin{aligned}
& \int_{D} \frac{\partial y}{\partial t}(y-v) d x+\int_{D} \nabla y \cdot \nabla(y-v) d x \leq \int_{D} f(y-v) d x \\
& \forall v \in L^{2}\left(0, T ; H_{0}^{1}(D)\right), v(t) \in \mathcal{K}(t) \text { a.e. }[0, T] . \\
& \int_{0}^{T} \int_{D} \frac{\partial y}{\partial t}(y-w) d x d t+\int_{0}^{T} \int_{D} \nabla y \cdot \nabla(y-w) d x d t \\
& \leq \int_{0}^{T} \int_{D} f(y-w) d x d t, \forall w \in \mathcal{K} .
\end{aligned}
$$

To (15), respectively (16), the initial condition:

$$
y(0, x)=y_{0}(x) \in \mathcal{K}(0) \subset H_{0}^{1}(D) \text { a.e. in } D,
$$

should be added.

It is known that, under the above compatibility and regularity assumptions, the parabolic variational inequalities (13), (15), (17) and (14), (16), (17) are equivalent and have a unique solution

$y \in L^{2}\left(0, T ; H^{2}(D)\right) \cap H^{1}\left(0, T ; L^{2}(D)\right)$ satisfying $y(t, x) \geq \psi(t, x)$ a.e. in $[0, T] \times D,[10]$.

Then, the above parabolic variational inequalities may be written in the form

$$
\frac{\partial y}{\partial t}-\Delta y+\beta(y-\psi) \ni f \text { a.e. in }[0, T] \times D
$$

together with (17), where the maximal monotone operator $\beta \subset \mathbb{R} \times \mathbb{R}$ is given by (8). Relation (18) is the strong formulation of the parabolic obstacle problem and makes sense due to the regularity of the solution $y \in L^{2}\left(0, T ; H^{2}(D)\right) \cap$ $H^{1}\left(0, T ; L^{2}(D)\right)$. The nonlinear term $\beta(y-\psi)$ should be understood as the "section" of the multivalued operator (8) occurring in (18).

We introduce two algorithms for the solution of (18), (17) that extend the method used for elliptic obstacle problems to the parabolic case (recall that $\beta_{\epsilon}$ is the regularization of $\beta$ ):

\section{Algorithm 2}

1) Choose $n=0, \epsilon_{0}>0, y_{-1}(t, x)=y_{0}(x), \tilde{y}_{-1}(t, x)=y_{0}(x)$.

2) Compute $y_{n} \in L^{2}\left(0, T ; H^{2}(D)\right) \cap H^{1}\left(0, T ; L^{2}(D)\right)$ as solution of the linear parabolic equation

$$
\begin{aligned}
\frac{\partial y_{n}}{\partial t}-\Delta y_{n}+\left[\beta_{\epsilon_{n}}^{\prime}\left(y_{n-1}-\psi\right)\right]\left(y_{n}-\psi\right) & =f, \text { a.e. in }[0, T] \times D, \\
y_{n}(0, x) & =y_{0}(x) \text { in } D .
\end{aligned}
$$


3) $\tilde{y}_{n}=\max \left(y_{n}, \psi\right), \epsilon_{n+1}=\frac{1}{2} \epsilon_{n}$

4) If $\left\|\tilde{y}_{n}-\tilde{y}_{n-1}\right\|_{L^{2}([0, T] \times D)}<$ tol then STOP; else $\mathrm{n}=\mathrm{n}+1$ GO TO step 2.

Since for the numerical solution of (linear or nonlinear) parabolic equations a time discretization has to be performed, a second algorithm may be formulated, that is close to the elliptic case. Namely, we consider Euler backward time discretization of (18) in the form:

$$
\frac{y^{k+1}-y^{k}}{\Delta t}-\Delta y^{k+1}+\beta\left(y^{k+1}-\psi\right)=f^{k+1} \text { in } D,
$$

with $y^{0}(x)=y_{0}(x)$ a.e. in $D$ and $f^{k+1}$ being an approximation of $f$ in $L^{2}([0, T] \times$ $D)$, (for instance $f^{k}(x)=f(k \Delta t, x)$ if $f$ is pointwisely defined).

Here $\Delta t=\frac{T}{m}, m \in \mathbb{N}^{*}$ and $k=0,1, \ldots, m-1$ in (19).

Remark 2.5. The discretization (19) is in fact also used in step 2) of Algorithm 2 , adapted to the linear parabolic equation defined there. By applying to (19) the corresponding Algorithm 1 for elliptic variational inequalities, we obtain

\section{Algorithm 3}

0) Put $y^{0}(x)=y_{0}(x)$.

For $k=0,1, \ldots, m-1, \Delta t=T / m$ do step 1) to step 4)

1) Choose $n=0, \epsilon_{0}>0, \tilde{y}_{-1}^{k+1}=y^{k}$

2) Compute $y_{n}^{k+1} \in H_{0}^{1}(D)$ as solution of the linear equation

$$
\frac{y_{n}^{k+1}-y^{k}}{\Delta t}-\Delta y_{n}^{k+1}+\left[\beta_{\epsilon_{n}}^{\prime}\left(y_{n-1}^{k+1}-\psi\right)\right]\left(y_{n}^{k+1}-\psi\right)=f^{k+1}
$$

3) $\tilde{y}_{n}^{k+1}=\max \left(y_{n}^{k+1}, \psi\right), \epsilon_{n+1}=\frac{1}{2} \epsilon_{n}$

4) If $\left\|\tilde{y}_{n}^{k+1}-\tilde{y}_{n-1}^{k+1}\right\|_{L^{2}(D)}<$ tol then $y^{k+1}=\tilde{y}_{n}^{k+1}$, STOP;

else $n=n+1$ GO TO step 2 .

Remark 2.6. In Algorithm 3, it is assumed that the time discretization of $[0, T]$ in $m$ equals subintervals is given and the starting point for the loop step 1) - step 4) is $y^{k}$ the value corresponding to $t=k \Delta t$ already computed at the previous time step.

We end this section with the two-phase Stefan problem and the corresponding algorithm (of the same type as Algorithm 3). Namely, we refer directly to the semidiscretized in time, two-phase Stefan problem, using the enthalpy formulation ([10]):

$$
\frac{\gamma\left(y^{k+1}\right)-\gamma\left(y^{k}\right)}{\Delta t}-\Delta y^{k+1}=f^{k+1} \text { in } D,
$$


Relation (20) corresponds to the implicit Euler scheme, $k=0, \ldots, m-1, y_{0}$ and $v_{0} \in \gamma\left(y^{0}\right)=\gamma\left(y_{0}\right)$ are given initial conditions for the temperature and the enthalpy, such that $y_{0} \in H_{0}^{1}(D), v_{0} \in L^{2}(D)$.

The maximal monotone enthalpy graph $\gamma \subset \mathbb{R} \times \mathbb{R}$

$$
\gamma(r)= \begin{cases}a r, & r<0 \\ {[0, L],} & r=0 \\ b r+L, & r>0\end{cases}
$$

with $a, b, L$ positive constants related to the thermal conductivities in the liquid/solid phases and to the latent heat. Notice that $\gamma(\cdot)$ has very different structure compared to (8) and there are no constraints of the type (13), (14).

The subsequent regularization $\gamma_{\epsilon}$ in (22) is similar to the Yosida regularization and it is Lipschitz continuous of constant $\frac{1}{\epsilon}$ :

$$
\gamma_{\epsilon}(r)= \begin{cases}a r, & r \leq 0 \\ \frac{1}{\epsilon} r, & 0<r<\frac{L \epsilon}{1-\epsilon b}, \\ b r+L, & r \geq \frac{L \epsilon}{1-\epsilon b} .\end{cases}
$$

Regularization and/or time discretization are standard techniques in the approximation of parabolic variational inequalities [4], [10], [12].

\section{Algorithm 4}

0) Choose $y^{0}(x)=y_{0}(x), \epsilon>0$.

For $k=0,1, \ldots, m-1$ do step 1) to step 3)

1) Choose $n=0, y_{-1}^{k+1}=y^{k}$.

2) Compute $y_{n}^{k+1} \in H_{0}^{1}(D)$ as solution of the linear parabolic equation

$$
\frac{\widetilde{\gamma}_{\epsilon}\left(y_{n}^{k+1}\right)-\gamma_{\epsilon}\left(y^{k}\right)}{\Delta t}-\Delta y_{n}^{k+1}=f^{k+1}, \text { in } D
$$

where

$$
\widetilde{\gamma}_{\epsilon}\left(y_{n}^{k+1}(x)\right)= \begin{cases}a y_{n}^{k+1}(x), & y_{n-1}^{k+1}(x) \leq 0, \\ \frac{1}{\epsilon} y_{n}^{k+1}(x), & 0<y_{n-1}^{k+1}(x)<\frac{L \epsilon}{1-\epsilon b}, \\ b y_{n}^{k+1}(x)+L, & y_{n-1}^{k+1}(x) \geq \frac{L \epsilon}{1-\epsilon b} .\end{cases}
$$

3) If $\left\|y_{n}^{k+1}-y_{n-1}^{k+1}\right\|_{L^{2}(D)}<$ tol then $y^{k+1}=y_{n}^{k+1}$, STOP; else $\mathrm{n}=\mathrm{n}+1$ GO TO step 2 .

Remark 2.7. In Algorithm 4, $\epsilon>0$ is fixed, small enough. It is in this way that the computations are performed, based on the known convergence properties for 
$\epsilon \rightarrow 0$ of the semidiscretized problem, [10]. In step 2), $\gamma_{\epsilon}\left(y^{k}\right)$ is computed after the relation (22) and $y^{k}$ is the obtained solution in the previous time step, after convergence. However $\widetilde{\gamma}_{\epsilon}\left(y_{n}^{k+1}\right)$ is computed by a similar formula, taking into account as well the values of the previous iteration $y_{n-1}^{k+1}$. Namely

$$
\widetilde{\gamma}_{\epsilon}\left(y_{n}^{k+1}\right)=\chi_{1} a y_{n}^{k+1}+\chi_{2} \frac{1}{\epsilon} y_{n}^{k+1}+\chi_{3}\left(b y_{n}^{k+1}(x)+L\right)
$$

where $\chi_{1}, \chi_{2}, \chi_{3}$ are the characteristic functions of $\left\{x \in D ; y_{n-1}^{k+1}(x) \leq 0\right\}$, $\left\{x \in D ; 0<y_{n-1}^{k+1}(x)<\frac{L \epsilon}{1-\epsilon b}\right\},\left\{x \in D ; y_{n-1}^{k+1}(x) \geq \frac{L \epsilon}{1-\epsilon b}\right\}$, respectively. This gives the linear character of the scheme, of the equation to be solved in each iteration. This is also valid in the previous algorithms.

\section{Stability and convergence}

\subsection{Elliptic case}

The fixed domain character of Algorithm 1 is very helpful for its analysis as well. We prove both the convergence of the solutions and of the geometry on a subsequence under general assumptions. If certain conditions are added, we show that the limit satisfies (1)-(4).

The following two lemmas show that the obstacle problem may be reduced to the case $\psi_{\mid \partial D}=0$, in general.

Lemma 3.1. Denote by $\hat{y} \in H^{2}(D) \cap H_{0}^{1}(D)$ the solution of

$$
-\Delta \hat{y}=f \text { in } D, \hat{y}=0 \text { on } \partial D .
$$

Then $y \geq \hat{y}$ a. e. in $D$, where $y$ is the solution of (1)-(4).

Proof. It is known that $y$ satisfies $y \in H^{2}(D) \cap H_{0}^{1}(D)$ and (1)-(4) can be equivalently expressed in the language of multivalued equations as

$$
-\Delta y+\beta(y-\psi)=f \text { a.e in } D, y=0 \text { on } \partial D .
$$

where $\beta \subset \mathbb{R} \times \mathbb{R}$ is the maximal monotone graph (8).

By regularity, the section of the multivalued function that occurs in (24) satisfied $\beta(y-\psi) \in L^{2}(D)$. Clearly, (8) shows that $\beta(y-\psi) \leq 0$ a.e. in $D$. Then a simple comparison argument for (23), (24) ends the proof.

Denote $\widehat{\psi}(x)=\max \{\psi(x), \hat{y}(x)\}$ in $D$. Then $\widehat{\psi} \in H_{0}^{1}(D)$ and $y(x) \geq \widehat{\psi}(x)$ a.e. in $D$. 
Lemma 3.2. The solution y of (1)-(4) satisfies the same problem with $\psi$ replaced by $\widehat{\psi}$.

Proof. We denote $\hat{K}=\left\{v \in H_{0}^{1}(D) ; v(x) \geq \widehat{\psi}(x)\right.$ a.e. in $\left.D\right\}$. By Lemma 3.1, $y \in \hat{K}$ and $\Delta y+f \leq 0$ a.e. in $D$ and it equals 0 if $y>\psi$ by (24).

For any $v \in \hat{K}$, we compute

$$
\begin{array}{r}
\int_{D}(\Delta y+f)(v-y) d x=\int_{D}(\Delta y+f)(\widehat{\psi}-y) d x \\
+\int_{D}(\Delta y+f)(v-\widehat{\psi}) d x \leq \int_{D}(\Delta y+f)(\widehat{\psi}-y) d x=0
\end{array}
$$

The last equality is a consequence of (1)-(2) and the fact that $y(x)=\psi(x)$ means $\hat{y} \leq \psi(x)$ and, consequently, $y(x)=\widehat{\psi}(x)$.

Integrating by parts in the first term of the above inequality, we get

$$
\int_{D} \nabla y \cdot(\nabla y-\nabla v) d x \leq \int_{D} f(y-v) d x, \forall v \in \hat{K} .
$$

This ends the proof.

Theorem 3.3. $i$ ) On a subsequence, $y_{n} \rightarrow \check{y}$ weakly in $H_{0}^{1}(D)$ and $\Omega_{n} \rightarrow \Omega$ in the complementary Hausdorff-Pompeiu topology, [26, Appendix 3].

ii) Assuming $\breve{y} \in \mathcal{C}^{1}(D)$ and $\left\{\Omega_{n}\right\}$ uniformly of class $\mathcal{C}$, then $\check{y}$ is the solution of (1)-(4) with $D^{+}=\Omega$ if the convergence is valid on the whole sequence.

Proof. i) By Lemma 3.1 and 3.2, we may assume $\psi=0$ on $\partial D$. Then $y_{n}-\psi$ is in $H_{0}^{1}(D)$ and may be used as test function in relation (9). We obtain

$$
\begin{aligned}
& \int_{D}\left|\nabla\left(y_{n}-\psi\right)\right|^{2} d x+\frac{1}{\epsilon_{n}} \int_{D \backslash \Omega_{n}}\left(y_{n}-\psi\right)^{2} d x \\
& =\int_{D} f\left(y_{n}-\psi\right) d x-\int_{D} \nabla \psi \cdot \nabla\left(y_{n}-\psi\right) d x .
\end{aligned}
$$

Then $\left\{y_{n}\right\}$ is bounded in $H_{0}^{1}(D)$ and

$$
\frac{1}{\epsilon_{n}} \int_{D \backslash \Omega_{n}}\left(y_{n}-\psi\right)^{2} d x \leq \text { const }, \forall n \in \mathbb{N} .
$$

We denote by $\check{y} \in H_{0}^{1}(D)$ the weak limit of $y_{n}$ in $H_{0}^{1}(D)$ on a subsequence and by $\Omega$ the limit of $\Omega_{n}$ (again on a subsequence) in the complementary HausdorffPompeiu topology. 
ii) If we assume that $\left\{\Omega_{n}\right\}$ is a family of open sets satisfying the uniform $\mathcal{C}$ property (i.e. the boundaries $\partial \Omega_{n}$ can be locally represented as graphs of uniformly continuous mappings), then it is known that $D \backslash \bar{\Omega}_{n} \rightarrow D \backslash \bar{\Omega}$ in the Hausdorff-Pompeiu complementary metric too.

Let $C \subset D \backslash \bar{\Omega}$ be any compact subdomain. Then $C \subset D \backslash \bar{\Omega}_{n}$, for $n \geq n_{C}$, by the $\Gamma$ property of the Hausdorff-Pompeiu complementary convergence, [26]. By (26), we get

$$
\frac{1}{\epsilon_{n}} \int_{C}\left(y_{n}-\psi\right)^{2} d x \leq \text { const, } \forall n \geq n_{C} .
$$

Then $\check{y}_{\mid C}=\psi$ a.e. in $C$. By letting $C \rightarrow D \backslash \bar{\Omega}$, we obtain $\check{y}_{\mid D \backslash \Omega}=\psi$ a.e. in $D \backslash \Omega$ (here we also use that the boundary of an open set of class $\mathcal{C}$ has zero Lebesgue measure, [26]).

Take now any $\rho \in \mathcal{C}_{0}^{\infty}(\Omega)$. Again by the $\Gamma$ property, we have that supp $\rho \subset \Omega_{n}$ for $n \geq n_{\rho}$. Consequently, $\rho$ may be used as test function on (9), for $n \geq n_{\rho}$ and we may let $n \rightarrow \infty$. We obtain that

$$
\int_{D} \nabla \check{y} \cdot \nabla \rho d x=\int_{D} f \rho d x .
$$

As supp $\rho \subset \Omega$ the integrals are in fact defined on $\Omega$ and we obtain that

$$
-\Delta \check{y}=f \text { in } \Omega
$$

in the sense of distributions.

We also notice that $\breve{y}-\psi \in H_{0}^{1}(\Omega)$ since $\check{y}-\psi=0$ a.e. in $D \backslash \Omega$ and domains of class $\mathcal{C}$ have the Keldysh-Hedberg stability property, [26]. This is related to the first part in (3). We underline that for domains of class $\mathcal{C}$, the usual trace theorems cannot be applied and the above relation on $(\partial \Omega) \cap D$ has to be carefully interpreted [26, Ch. 2].

Finally, let $Q \subset \Omega$ be any compact subdomain. Again by the $\Gamma$-property of the Hausdorff-Pompeiu complementary convergence for the open sets, we get $Q \subset$ $\Omega_{n}$, for $n \geq n_{Q}$. Then, $y_{n}(x)>\psi(x)$ for $x \in Q, n \geq n_{Q}$. By $y_{n} \rightarrow \breve{y}$ strongly in $L^{2}(D)$, we infer $\breve{y}(x) \geq \psi(x)$ a.e. in $Q$. Consequently, $\breve{y}(x) \geq \psi(x)$ a.e. in $\Omega$. The points on $\partial \Omega \backslash \partial D$, are minimum points for $\check{y}-\psi$. Under $\mathcal{C}^{1}$ regularity assumptions, we obtain $\nabla(\check{y}-\psi)=0$ on $\partial \Omega \backslash \partial D$, that is (3) is satisfied for $D^{+}=\Omega$.

Concerning relation (2), we consider again $C$ as above and we notice that $y_{n} \leq$ $\psi$ a.e. in $C$ for $n \geq n_{C}$, due to step 3 in Algorithm 1. Then, we obtain (2) in $C$, due to the positivity of the penalized coefficient. One can pass to the limit (with $n$ and $C$ ) in the sense of distributions. 
Remark 3.4. If $f \in L^{\infty}(D)$, then $y \in W^{2, p}(D)$ for any $p \geq 1$. By the Sobolev theorem, we get $y \in \mathcal{C}^{1}(D)$ in arbitrary dimension. Regularity results for the solution of the variational inequality and for the free boundary may be found in [20] and [22]. This justifies the regularity assumption.

Remark 3.5. The above argument shows that the sequences constructed in Algorithm 1 have accumulation points. Under supplementary assumptions, the limit point gives the solution of (1)-(4). The algorithm has a constructive character both for the functions and the geometry.

\subsection{Parabolic case}

We discuss first Algorithm 2. Denote by $\widehat{y} \in W^{1,2}\left(0, T ; L^{2}(D)\right) \cap L^{2}\left(0, T ; H^{2}(D)\right)$, the unique solution of the linear parabolic equation

$$
\begin{aligned}
\frac{\partial \widehat{y}}{\partial t}-\Delta \widehat{y} & =f, \text { a.e. in }(0, T) \times D, \\
\widehat{y}(t, x) & =0, \text { in }[0, T] \times \partial D, \\
\widehat{y}(0, x) & =y_{0}(x), \text { in } D .
\end{aligned}
$$

Let $\widehat{\psi}=\max (\psi, \widehat{y}) \in H^{1}\left(0, T ; L^{2}(D)\right) \cap L^{2}\left(0, T ; H_{0}^{1}(D)\right)$, that is $\widehat{\psi}$ has null traces on $\partial D$; and let

$$
\widehat{\mathcal{K}}=\left\{v \in L^{2}\left(0, T ; H_{0}^{1}(D)\right) ; v(t, x) \geq \widehat{\psi}(t, x) \text { a.e. }[0, T] \times D\right\} .
$$

Lemma 3.6. The unique solution $y \in W^{1,2}\left(0, T ; L^{2}(D)\right) \cap L^{2}\left(0, T ; H^{2}(D)\right)$ of (16) satisfies as well the same variational inequality with $\mathcal{K}$ replaced by $\widehat{\mathcal{K}}$.

Proof. The variational inequality (16) may be rewritten as (18), due to the regularity properties. By (8), we have that $\beta(y-\psi) \leq 0$ a.e. in $[0, T] \times D$ (the section that occurs in (18) and belongs to $L^{2}([0, T] \times D)$ ).

A simple comparison argument for (18) and (28)-(30) shows that $y \geq \widehat{y}$ a.e. in $[0, T] \times D$. It yields that $y \geq \widehat{\psi}$ a.e. in $[0, T] \times D$. We also have

$$
\frac{\partial y}{\partial t}-\Delta y-f \geq 0 \text { a.e. in }[0, T] \times D
$$

due to (8), (18). 
We can estimate, for any $w \in \widehat{\mathcal{K}}$ :

$$
\begin{array}{r}
\int_{0}^{T} \int_{D} \frac{\partial y}{\partial t}(y-w) d x d t-\int_{0}^{T} \int_{D} \Delta y(y-w) d x d t \\
-\int_{0}^{T} \int_{D} f(y-w) d x d t \geq \int_{0}^{T} \int_{D} \frac{\partial y}{\partial t}(y-\widehat{\psi}) d x d t \\
-\int_{0}^{T} \int_{D} \Delta y(y-\widehat{\psi}) d x d t-\int_{0}^{T} \int_{D} f(y-\widehat{\psi}) d x d t=0
\end{array}
$$

due to (32), (31).

The last equality in (33) comes from the remark that $y=\psi \Rightarrow \widehat{y} \leq \psi \Rightarrow$ $y=\widehat{\psi}$ as a pointwise relation valid a.e. on the coincidence set associated to (18). Then, the last equality in (33) is a consequence of the formulation of (16) as a complementarity relations problem.

Remark 3.7. By Lemma 3.6, we may assume that the obstacle $\psi$ has null traces on $\partial D$. Notice that $\widehat{\psi}$ doesn't satisfy $\widehat{\psi} \in L^{2}\left(0, T ; H^{2}(D)\right)$ but this is not important in the sequel.

Proposition 3.8. We have $\left\{y_{n}\right\}$ bounded in $L^{\infty}\left(0, T ; L^{2}(D)\right) \cap L^{2}\left(0, T ; H_{0}^{1}(D)\right)$ and $y_{n} \rightarrow \widetilde{y}$ on a subsequence weakly in $L^{\infty}\left(0, T ; L^{2}(D)\right) \cap L^{2}\left(0, T ; H_{0}^{1}(D)\right)$. Moreover, if $y$ is the solution of (14), (16), (17), then $\widetilde{y} \leq y$ a.e. in $[0, T] \times D$ and

$$
\int_{Q_{n}}\left(y_{n}-\psi\right)^{2} d x d t \leq C \epsilon_{n}
$$

with $C$ independent of $n$ and $Q_{n}=\left\{(t, x) \in[0, T] \times D ; y_{n-1}(t, x)<\psi(t, x)\right\}$.

Proof. In Step 2, Algorithm 2, we multiply the equation by $y_{n}-\psi \in H^{1}\left(0, T ; L^{2}(D)\right) \cap L^{2}\left(0, T ; H_{0}^{1}(D)\right)$. After partial integration, we get

$$
\begin{aligned}
& \frac{1}{2}\left\|y_{n}(t)\right\|_{L^{2}(D)}^{2}-\frac{1}{2}\left\|y_{0}\right\|_{L^{2}(D)}^{2}-\int_{0}^{T} \int_{D} \frac{\partial y_{n}}{\partial t} \psi d x d t+ \\
& +\int_{0}^{T} \int_{D}\left|\nabla y_{n}\right|^{2} d x d t-\int_{0}^{T} \int_{D} \nabla y_{n} \cdot \nabla \psi d x d t \\
& \leq \int_{0}^{T} \int_{D} f\left(y_{n}-\psi\right) d x d t
\end{aligned}
$$


since the last term on the left-hand side of the equation, multiplied by $\left(y_{n}-\psi\right)$, is positive. The first integral in (34) may be written as

$$
\begin{aligned}
& \int_{0}^{t} \int_{D} \frac{\partial y_{n}}{\partial t} \psi d x d t=\int_{D} y_{n}(t, x) \psi(t, x) d x- \\
& -\int_{D} y_{0}(x) \psi(0, x) d x-\int_{0}^{t} \int_{D} y_{n} \frac{\partial \psi}{\partial t} d x d t
\end{aligned}
$$

Combining (34), (35) we get $\left\{y_{n}\right\}$ bounded in $L^{\infty}\left(0, T ; L^{2}(D)\right) \cap L^{2}\left(0, T ; H_{0}^{1}(D)\right)$.

In (34), (35), we have neglected the positive and bounded term

$$
\int_{0}^{t} \int_{D}\left[\beta_{\epsilon_{n}}^{\prime}\left(y_{n}-\psi\right)\right]\left(y_{n}-\psi\right)^{2} d x d t \leq C, \forall t \in[0, T]
$$

where $C$ is independent of $n$.

We fix $t=T$ in (36) and taking into account the definition of $Q_{n}$, we get

$$
\int_{Q_{n}}\left(y_{n}-\psi\right)^{2} d x d t \leq C \epsilon_{n}, \forall t \in[0, T]
$$

due to the form of $\beta_{\epsilon_{n}}(\cdot)$.

We have the inequality:

$$
\begin{aligned}
& {\left[\beta_{\epsilon_{n}}^{\prime}\left(y_{n}-\psi\right)\right]\left(y_{n}-\psi\right)=\left[\beta_{\epsilon_{n}}^{\prime}\left(y_{n-1}-\psi\right)\right]\left(y_{n-1}-\psi\right)+} \\
& +\left[\beta_{\epsilon_{n}}^{\prime}\left(y_{n-1}-\psi\right)\right]\left(y_{n}-\psi-y_{n-1}+\psi\right) \geq \beta_{\epsilon_{n}}\left(y_{n-1}-\psi\right)+ \\
& +\beta_{\epsilon_{n}}\left(y_{n}-\psi\right)-\beta_{\epsilon_{n}}\left(y_{n-1}-\psi\right)=\beta_{\epsilon_{n}}\left(y_{n}-\psi\right)
\end{aligned}
$$

In (38), we use $\beta_{\epsilon_{n}}^{\prime}(r) r=\beta_{\epsilon_{n}}(r)$, for all $r \in \mathbb{R}$ (it is valid for $r=0$ as well, although in $r=0, \beta_{\epsilon_{n}}$ has just two finite lateral derivative) and the definition of the concave subdifferential since $\beta_{\epsilon_{n}}$ is a concave continuous function.

By (38) and Step 2 of Algorithm 2, we infer

$$
\frac{\partial y_{n}}{\partial t}-\Delta y_{n}+\beta_{\epsilon_{n}}\left(y_{n}-\psi\right) \leq f, \text { a.e. in }[0, T] \times D \text {. }
$$

Denote by $z_{n} \in L^{2}\left(0, T ; H^{2}(D)\right) \cap H_{0}^{1}(D) \cap W^{1,2}\left(0, T ; L^{2}(D)\right)$ the unique solution of

$$
\begin{aligned}
& \frac{\partial z_{n}}{\partial t}-\Delta z_{n}+\beta_{\epsilon_{n}}\left(z_{n}-\psi\right)=f, \text { a.e. in }[0, T] \times D \\
& z_{n}(0, x)=y_{0}(x), \text { a.e. in } D .
\end{aligned}
$$


It is known that $z_{n} \rightarrow y$ weakly in $L^{2}\left(0, T ; H^{2}(D)\right) \cap W^{1,2}\left(0, T ; L^{2}(D)\right)$ and strongly in $\mathcal{C}\left(0, T ; L^{2}(D)\right)$, with $y$ the solution of the variational inequality (16), (17).

A simple comparison argument between (39) and (40) shows that

$$
y_{n}(t, x) \leq z_{n}(t, x), \text { a.e. in }[0, T] \times D .
$$

On a subsequence, we may assume that $y_{n} \rightarrow \widetilde{y}$ weakly in $L^{\infty}\left(0, T ; L^{2}(D)\right) \cap L^{2}\left(0, T ; H_{0}^{1}(D)\right)$ and (42) gives $\widetilde{y} \leq y$ a.e. in $[0, T] \times D$.

Remark 3.9. The inequality (37) says that $y_{n}$ is above the obstacle $\psi$, asymptotically. Under regularity conditions (for instance, if $Q_{n}$ are open and uniformly of class $\mathcal{C}$, [26]) one can study their limit $Q$ in the Hausdorff-Pompeiu complementary metric, on a subsequence, and show that $\widetilde{y}=\psi$ in $Q$. In the numerical examples, stronger convergence properties of $\left\{y_{n}\right\}$ to the solution of (16)-(17) are observed. For the Algorithm 3, the elliptic iterations are discussed in Theorem 3.3, while the convergence of the parabolic semidiscretization is proved in [10].

For the Algorithm 4, we establish the following properties.

Proposition 3.10. $i)$ The sequence $\left\{y_{n}^{k+1}\right\}$ is bounded in $H_{0}^{1}(D)$ with respect to n. If $y_{0} \in H_{0}^{1}(D) \cap L^{p}(D)$ and $f$ is in $\mathcal{C}\left(0, T ; L^{p}(D)\right), p \geq 2$, then $\left\{y_{n}^{k+1}\right\}$ is bounded in $W^{2, p}(D) \cap H_{0}^{1}(D)$ with respect to $n$ (and weakly convergent on a subsequence).

ii) If $y_{n}^{k+1} \rightarrow \widetilde{y}^{k+1}$ weakly in $W^{2, p}(D), p>d$, then $\widetilde{y}^{k+1}$ satisfies

$$
\frac{\gamma_{\epsilon}\left(\widetilde{y}^{k+1}\right)-\gamma_{\epsilon}\left(y^{k}\right)}{\Delta t}-\Delta \widetilde{y}^{k+1}=f^{k+1} \text {, in } D .
$$

Proof. i) Notice that by the definition of $\widetilde{\gamma}_{\epsilon}$ (that ensures the equation in Step 2 Algorithm 4 to be piecewise linear), it may happen that $y_{n}^{k+1}=0$ in certain points, but $\widetilde{\gamma}_{\epsilon}\left(y_{n}^{k+1}\right) \neq 0$ in that points. However, $\widetilde{\gamma}_{\epsilon}$ remains a sublinear mapping.

Multiplying the equation by $y_{n}^{k+1}$ and integrating by parts, we infer $\left\{y_{n}^{k+1}\right\}$ bounded in $H_{0}^{1}(D)$ with respect to $n$ as claimed.

Under the supplementary $L^{p}(D)$ assumptions, the conclusion follows by the $L^{p}(D)$ theory for elliptic variational inequalities, using induction with respect to $k$ (the boundedness constant may depend on $k$ ) and the sublinearity of $\widetilde{\gamma}_{\epsilon}$. One may take subsequences with $y_{n}^{k+1} \rightarrow \widetilde{y}^{k+1}$ weakly in $W^{2, p}(D)$.

ii) If the convergence is valid on the whole sequence, weakly in $W^{1, p}(D)$, $p>d$, then $y_{n}^{k+1}(x) \rightarrow \widetilde{y}^{k+1}(x)$ uniformly in $\bar{D}$, for $n \rightarrow \infty$, without taking subsequences. One can pass to the limit in the definition of $\widetilde{\gamma}_{\epsilon}$ and $\widetilde{\gamma}_{\epsilon}\left(y_{n}^{k+1}(x)\right) \rightarrow$ $\gamma_{\epsilon}\left(\widetilde{y}^{k+1}(x)\right)$. Then, by Step 2 of Algorithm 4, we get the conclusion. 
Remark 3.11. We write $\widetilde{y}^{k+1}=y^{k+1}$. A similar property may be stated in connection with Proposition 3.8 as well.

Remark 3.12. On a subsequence, we may assume that $\lim _{n \rightarrow \infty} y_{n}^{k+1}=\widetilde{y}^{k+1}$. However, it is not clear how to pass to the limit in $\widetilde{\gamma}_{\epsilon}\left(y_{n}^{k+1}\right)$ since $y_{n-1}^{k+1}$ may be (in principle) convergent on a different subsequence and to a different limit. However, in the numerical experiments, the test in Step 3 of Algorithm 4 is fulfilled, that is $\left\{y_{n}^{k+1}\right\}$ and $\left\{y_{n-1}^{k+1}\right\}$ have the same limit. Denote it by $y^{k+1} \in H_{0}^{1}(D)$. By passing to the limit, it would satisfy the equation (43). The analysis of (43) for $k \rightarrow \infty$ and $\epsilon \rightarrow 0$ is known [10], [33].

\section{Numerical tests}

We have used the software FreeFem++ v 3.12, [15].

\subsection{Elliptic case}

\section{Test 1.}

We use Algorithm 1 for solving (5)-(6).

We set $D=\left\{\left(x_{1}, x_{2}\right) ; \sqrt{x_{1}^{2}+x_{2}^{2}}<1\right\}$. The obstacle $\psi$ is given by the formula

$$
\psi\left(x_{1}, x_{2}\right)=\left\{\begin{array}{l}
\frac{1}{2}, \text { if } 0.5 \leq x_{1} \leq 0.7,-0.1 \leq x_{2} \leq 0.1 \\
-12.5\left(\left(x_{1}+0.4\right)^{2}+x_{2}^{2}\right)+0.5, \text { if }\left(x_{1}+0.4\right)^{2}+x_{2}^{2} \leq 0.08 \\
-\frac{1}{2}, \text { otherwise }
\end{array}\right.
$$

We notice that $\psi$ is not continuous, but the method still works.

Let $f \in L^{2}(D)$ given by

$$
f\left(x_{1}, x_{2}\right)= \begin{cases}3.5, & x_{1}^{2}+x_{2}^{2} \leq 0.16 \\ -0.001, & \text { otherwise }\end{cases}
$$

The mesh has 109898 triangles and 55350 vertices, and we use the fixed penalization parameter $\epsilon_{n}=10^{-4}$. The stopping test was $\left\|\mathbf{y}_{n}-\mathbf{y}_{n-1}\right\|_{L^{2}(D)}<$ tol $=$ $10^{-17}$ or $\left\|\mathbf{y}_{n}-\mathbf{y}_{n-1}\right\|_{H^{1}(D)}<$ tol $=10^{-14}$. Starting from $D \backslash \Omega_{0}=\emptyset$, the history of error of computed solution between two consecutive iterations is presented in Table 1.

After 11 iterations the relative error no longer changes. The coincidence set and the computed solution are shown in Figure 2. The convergence order in this example is superlinear (see [29]). 


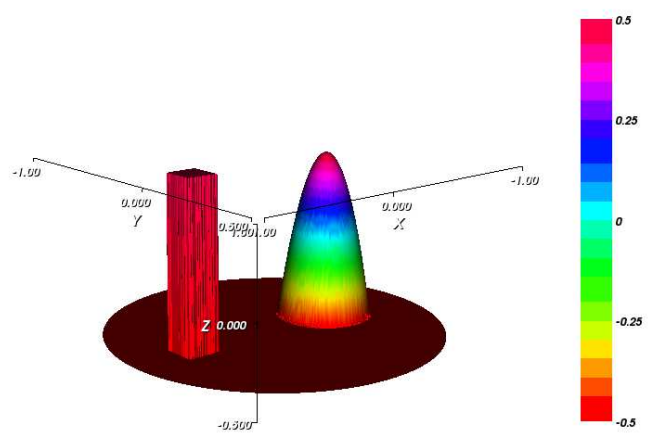

Figure 1. The obstacle for the Test 1.
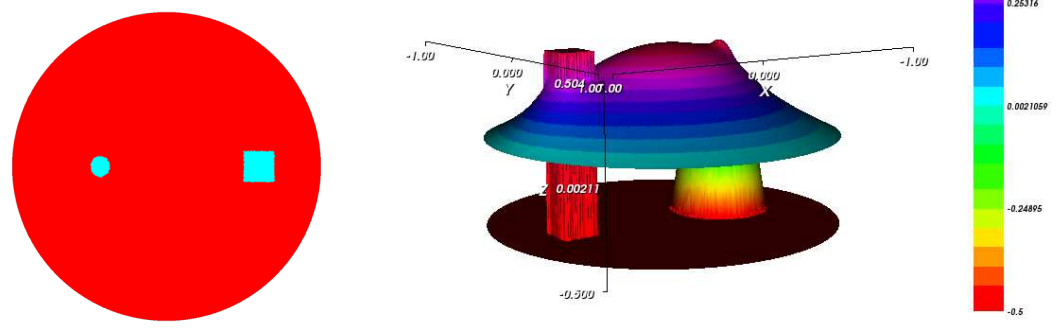

Figure 2. The coincidence set (blue) at the left and the computed solution with the obstacle for the Test 1.

We have tested the stability of the algorithm when $f$, the right-hand side in (5), is perturbed by $\delta f: D \rightarrow \mathbb{R}$ defined by

$$
\delta f\left(x_{1}, x_{2}\right)=\frac{k}{100}+\frac{k}{10} \sin \left(10 k x_{1}\right) \cos \left(10 k x_{2}\right)
$$

for $k \in\{1,2,3,4,5\}$. We denote the computed solution of (5)-(6) with the righthand side $f$ and $f+\delta f$ by $y_{h}$ and $y_{h}+\delta y_{h}$, respectively. The notation doesn't take into account the (nonlinear) dependence on $k$, for simplicity of writing. We observe that the second column of Table 2 is bounded, which shows that the convergence property proved in Theorem 3.3 is satisfied in this example. Similar com- 


\begin{tabular}{|c|c|c|c|c|c|c|}
\hline iteration & 0 & 2 & 4 & 6 & 8 & 10 \\
\hline$\left\|\mathbf{y}_{n}-\mathbf{y}_{n-1}\right\|_{L^{2}(D)}$ & 0.320 & 0.026 & 0.009 & 0.001 & $9.3 \mathrm{e}-5$ & $5.3 \mathrm{e}-18$ \\
\hline$\left\|\mathbf{y}_{n}-\mathbf{y}_{n-1}\right\|_{H^{1}(D)}$ & 4.213 & 0.274 & 0.106 & 0.025 & $1.9 \mathrm{e}-4$ & $1.0 \mathrm{e}-15$ \\
\hline
\end{tabular}

Table 1. History of the error for Test 1.

ment for the third column in Table 2. Moreover, the coincidence set associated to the perturbed solutions cannot be distinguished graphically from the unperturbed one represented in Figure 2 at the left.

\begin{tabular}{|c|c|c|c|}
\hline$k$ & $\left\|\delta y_{h}\right\|_{L^{2}(D)} /\|\delta f\|_{L^{2}(D)}$ & $\left\|\delta y_{h}\right\|_{L^{2}(D)} /\left\|y_{h}\right\|_{L^{2}(D)}$ & $\left\|\delta y_{h}\right\|_{L^{2}(D)}$ \\
\hline 1 & 0.0167631 & 0.00351167 & 0.00150832 \\
\hline 2 & 0.0166544 & 0.00699263 & 0.00300345 \\
\hline 3 & 0.0164874 & 0.01031800 & 0.00443176 \\
\hline 4 & 0.0166252 & 0.01380200 & 0.00592817 \\
\hline 5 & 0.0167180 & 0.01719910 & 0.00738730 \\
\hline
\end{tabular}

Table 2. Test 1 . Stability when $f$ is perturbed.

\section{Tests 2}

In [5], a level set method with artificial time variable is discussed which approximates the steady obstacle problem. We consider the second and the third examples from [5]. Since the considered boundary data are very close to the given obstacle (just slightly different in certain parts of the boundary) finding the coincidence set becomes a rather difficult task.

Test 2.1. The domain for the second example from [5] is:

$$
\begin{aligned}
& D=\left\{\left(x_{1}, x_{2}\right) ;\left(x_{1}-0.5\right)^{2}+\left(x_{2}-0.5\right)^{2} \leq 0.5^{2}, x_{2}>0.5\right\} \\
& \cup([0,1] \times[0.2,0.5]) \backslash([0.3,0.45] \times[0.2,0.45] \cup[0.6,0.75] \times[0.2,0.35]) .
\end{aligned}
$$

We set $f(x)=-1$ and $\psi(x)=0$, for all $x \in D$. We solve the problem (5), but we impose nonhomogenous boundary conditions

$$
K=\left\{v \in H^{1}(D) ; v \geq \psi \text { a.e. in } D, v=g \text { on } \partial D\right\}
$$


where

$$
g(x)= \begin{cases}0, & x_{2} \leq 0.5 \\ c \exp \left(-200\left(x_{1}-0.5\right)^{2}\right), & \text { otherwise. }\end{cases}
$$

Also, at the Step 2, Algorithm 1, the solution of the linear elliptic equation has nonhomogenous boundary conditions.

We use a mesh of 4874 triangles, 2555 vertices, the tolerance for the stopping test $t o l=10^{-7}$ and the penalization parameter is $\epsilon_{n}=0.003$. The Algorithm 1 stops after 4 iterations if $c=3$. In [5], the parameters $c=1, c=0.5$ are used, but in our algorithms the round-off errors influence the result if $c<3$.

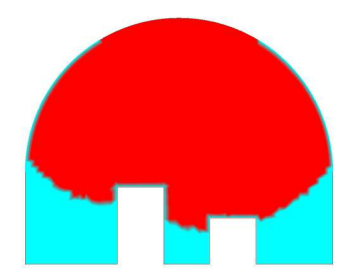

Figure 3. Test 2.1. Coincidence set (blue) for $c=3$.

Test 2.2. The domain $D$ for the third example from [5] is a segment of a circle with radius 1 , opening angle $\pi / 3$ and center $(0,0)$. As in the Test 2.1, we set $f(x)=-1$ and $\psi(x)=0$. We solve the problem (5), but we impose nonhomogenous boundary conditions

$$
K=\left\{v \in H^{1}(D) ; v \geq \psi \text { a.e. in } D, v=g \text { on } \partial D\right\}
$$

where

$$
g\left(x_{1}, x_{2}\right)= \begin{cases}c \exp \left(-400\left(x_{1}-0.825\right)^{2}\right), & \text { if } x_{1}^{2}+x_{2}^{2}=1 \\ 0, & \text { otherwise }\end{cases}
$$

We use a mesh of 4278 triangles, 2230 vertices, the tolerance for the stopping test $t o l=10^{-20}$ and the penalization parameter is $\epsilon_{n}=0.003$. The coincidence set and the computed solution are presented in Figure 4. The boundary function $g$ takes strictly positive values on the circular boundary, but close to the corners, $g$ takes very small values, for example for $x_{1}=0.98, g$ is of order $10^{-5}$. For this reason, the computed coincidence set contains the parts of the circular boundary close to the corners. The value of the parameter $c=0.5$ and the numerical results are comparable with [5]. 

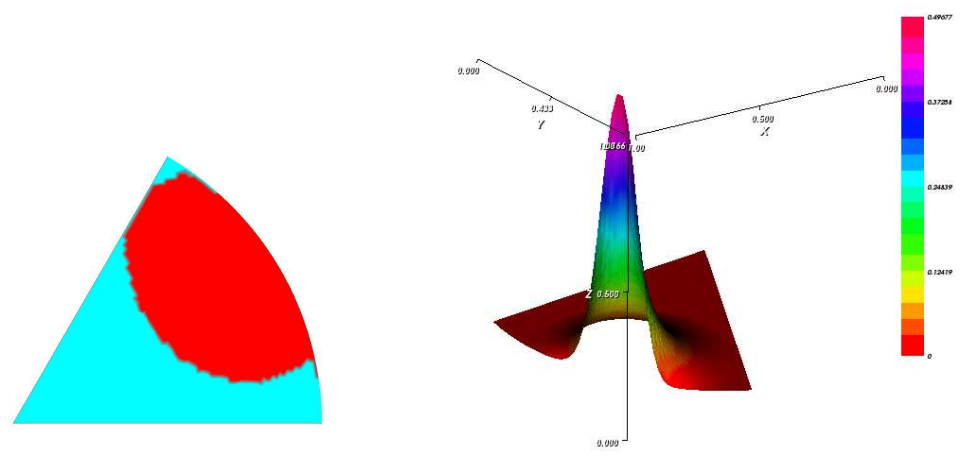

Figure 4. Test 2.2. The coincidence set (blue) at the left and the computed solution.

The number of (artificial) time advancing iterations for the level set method from [5] is greater than 60, see Figure 3, (f) from [5], while the Algorithm 1 stops after 6 iterations.

\subsection{Parabolic case}

\section{Test 3. One phase Stefan problem}

We have adapted the two-dimensional melting problem from [10], p. 148. The problem can be written as (18), (8), where $D=(-1,1) \times(-1,1), T=0.5, \psi=0$ and $f=-2$ on $[0, T] \times D$.

We have used the tolerance for the stopping test $t o l=10^{-6}$ in Algorithm 3 and tol $=N \times 10^{-6}$ in Algorithm 2, where $N$ is the number of the time steps. We consider a fixed penalization parameter $\epsilon_{n}=10^{-3}$, for all $n \in \mathbb{N}$.

For the time step $\Delta t=0.05$ and the mesh size $h=1 / 160$, the error in the norm of $L^{2}\left(0, T ; L^{2}(D)\right)$ between the solutions obtained by the Algorithms 2 and 3 is 0.000056. The same error is 0.00104 in the norm of $L^{2}\left(0, T ; H^{1}(D)\right)$. They confirm the results indicated in [10]. The convergence of our method is fast, the Algorithm 2 ends after $n=10$ iterations, and the Algorithm 3 performs $n=6$ or $n=5$ iterations by time step.

\section{Test 4. Two phase Stefan problem}

We consider the example b) p. 88 from [10] for testing the Algorithm 4.

The domain is $D=\left\{\left(x_{1}, x_{2}\right) ; x_{1}^{2}+x_{2}^{2} \leq 1\right\}$, the final time is $T=0.5$, the 


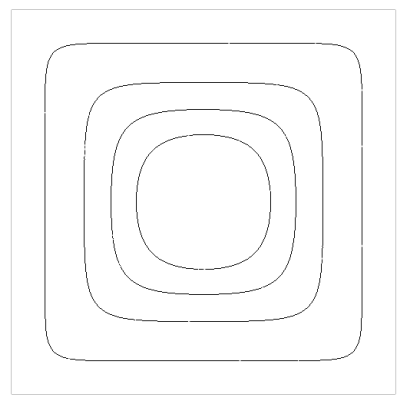

Figure 5. Test 3. The free boundary position at $t=0.05, t=0.15, t=0.30$, $t=0.5$ (from the exterior to the center)

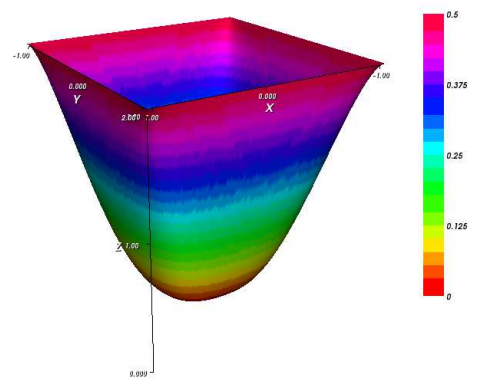

Figure 6. Test 3. Computed solution at $t=0.5$

right-hand side is

$$
f\left(t, x_{1}, x_{2}\right)= \begin{cases}8\left(2 e^{-2 t}-1\right), & \sqrt{x_{1}^{2}+x_{2}^{2}}>e^{-t}, \\ 2\left(2 e^{-2 t}-2\right), & \sqrt{x_{1}^{2}+x_{2}^{2}} \leq e^{-t},\end{cases}
$$

the enthalpy graph (21) has the form

$$
\gamma(r)= \begin{cases}r, & r<0 \\ {[0,2],} & r=0 \\ 4 r+2, & r>0\end{cases}
$$

and the initial condition is $y_{0}\left(x_{1}, x_{2}\right)=x_{1}^{2}+x_{2}^{2}-1$. 
The exact solution is

$$
y\left(t, x_{1}, x_{2}\right)= \begin{cases}2\left(x_{1}^{2}+x_{2}^{2}-e^{-2 t}\right), & \sqrt{x_{1}^{2}+x_{2}^{2}}>e^{-t}, \\ x_{1}^{2}+x_{2}^{2}-e^{-2 t}, & \sqrt{x_{1}^{2}+x_{2}^{2}} \leq e^{-t} .\end{cases}
$$

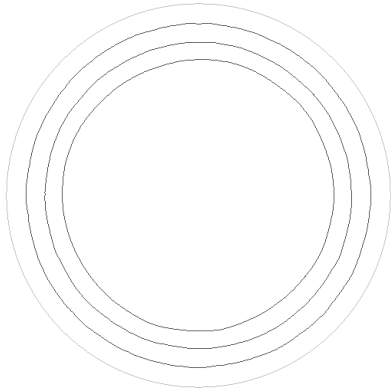

Figure 7. Test 4 . The free boundary position at $t=0.1, t=0.2, t=0.3$ (from the exterior to the center)

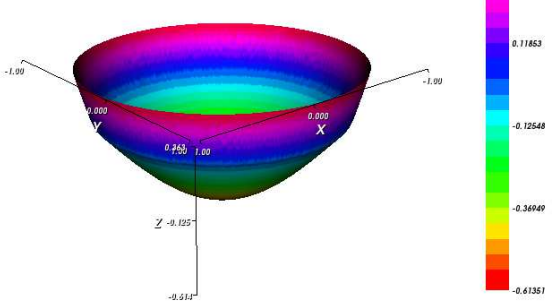

Figure 8. Test 4. Computed solution at $t=0.1$

We have applied the Algorithm 4 with a mesh of 85030 triangles and 42866 vertices, the mesh size is $h=0.017459$, the tolerance for the stopping test $t o l=$ $10^{-3}$ and a fixed penalization parameter $\epsilon_{n}=10^{-2}$, for all $n \in \mathbb{N}$.

The error between the exact and the calculated solution for the time step $\Delta t=$ 0.01 and the number of time steps $N=50$ is 0.193753 in the norm of $L^{2}\left(0, T ; L^{2}(D)\right)$ and 0.650485 in the norm of $L^{2}\left(0, T ; H^{1}(D)\right)$. 
The results indicate in this example that, at each time step, the Algorithm 4 converges linearly.

\section{Test 5. Two-dimensional Black-Scholes equation}

We consider an example from [30] with application to finance: the two-asset American put option: the right (not the obligation) to sell assets at a fixed price (exercise or strike price), before a given data (expire date).

The value of the option $V: \bar{D} \times[0, T] \rightarrow \mathbb{R}$ verifies the two-dimensional Black-Scholes equation

$$
\begin{aligned}
& \frac{\partial V}{\partial t}\left(S_{1}, S_{2}, t\right)+\mathcal{L} V\left(S_{1}, S_{2}, t\right) \leq 0, \text { in } D \times(0, T) \\
& \left(\frac{\partial V}{\partial t}\left(S_{1}, S_{2}, t\right)+\mathcal{L} V\left(S_{1}, S_{2}, t\right)\right)\left(\psi\left(S_{1}, S_{2}\right)-V\left(S_{1}, S_{2}, t\right)\right)=0 \\
& \text { in } D \times(0, T) \\
& V\left(S_{1}, S_{2}, t\right) \geq \psi\left(S_{1}, S_{2}\right), \text { in } D \times(0, T) \\
& V\left(S_{1}, S_{2}, T\right)=\psi\left(S_{1}, S_{2}\right), \text { in } D \\
& V\left(S_{1}, 0, t\right)=g_{1}\left(S_{1}, t\right), S_{1} \in[0, L], t \in(0, T) \\
& V\left(S_{1}, H, t\right)=0, S_{1} \in[0, L], t \in(0, T) \\
& V\left(0, S_{2}, t\right)=g_{2}\left(S_{2}, t\right), S_{2} \in[0, H], t \in(0, T) \\
& V\left(L, S_{2}, t\right)=0, S_{2} \in[0, H], t \in(0, T)
\end{aligned}
$$

where

$$
\begin{aligned}
\mathcal{L} V= & \left(r-\delta_{1}\right) S_{1} \frac{\partial V}{\partial S_{1}}+\left(r-\delta_{2}\right) S_{2} \frac{\partial V}{\partial S_{2}} \\
& +\frac{1}{2}\left(\left(\sigma_{1} S_{1}\right)^{2} \frac{\partial^{2} V}{\partial\left(S_{1}\right)^{2}}+2 \rho \sigma_{1} \sigma_{2} S_{1} S_{2} \frac{\partial^{2} V}{\partial S_{1} S_{2}}+\left(\sigma_{2} S_{2}\right)^{2} \frac{\partial^{2} V}{\partial\left(S_{2}\right)^{2}}\right)-r V,
\end{aligned}
$$

$T$ is the expire or maturity date, $S_{i}$ is the value of the $\mathrm{i}$-th asset, $i=1,2, \rho \in$ $[-1,0) \cup(0,1]$ is the correlation of two assets, $r$ is the risk-free interest rate, $\sigma_{i}$ is the volatility of the $\mathrm{i}$-th asset, $\delta_{i}$ represents the dividend of the $\mathrm{i}$-th asset.

The payoff function is $\psi: D=(0, L) \times(0, H) \rightarrow \mathbb{R}$

$$
\psi\left(S_{1}, S_{2}\right)=\min \left\{\left(K-S_{1}\right)^{+},\left(K-S_{2}\right)^{+}\right\}=\left(K-\max \left(S_{1}, S_{2}\right)\right)^{+},
$$

which corresponds to the American min-put contract, where $K>0$ is the strike price. Other payoff functions for two or more assets are discussed in [8]. 
This parabolic obstacle problem with terminal condition at $t=T$, can be rewritten as a parabolic problem with initial condition at $t=0$ and constant coefficients by a change of variables $S_{i}=e^{x_{i}}, \tau=T-t$.

Following [38], we can rewrite the operator $\mathcal{L}$ in a conservative form

$$
-\mathcal{L} V=-\nabla \cdot(A \nabla V+b V)+c V
$$

where

$$
\begin{aligned}
& A=\left(\begin{array}{ll}
a_{11} & a_{12} \\
a_{21} & a_{22}
\end{array}\right)=\left(\begin{array}{cc}
\frac{1}{2}\left(\sigma_{1} S_{1}\right)^{2} & \frac{1}{2} \rho \sigma_{1} \sigma_{2} S_{1} S_{2} \\
\frac{1}{2} \rho \sigma_{1} \sigma_{2} S_{1} S_{2} & \frac{1}{2}\left(\sigma_{2} S_{2}\right)^{2}
\end{array}\right) \\
& b=\left(\begin{array}{l}
b_{1} \\
b_{2}
\end{array}\right)=\left(\begin{array}{c}
\left(r-\delta_{1}\right) S_{1}-\left(\sigma_{1}\right)^{2} S_{1}-\frac{1}{2} \rho \sigma_{1} \sigma_{2} S_{1} \\
\left(r-\delta_{2}\right) S_{2}-\left(\sigma_{2}\right)^{2} S_{2}-\frac{1}{2} \rho \sigma_{1} \sigma_{2} S_{2}
\end{array}\right)
\end{aligned}
$$

and $c=3 r-\delta_{1}-\delta_{2}-\left(\left(\sigma_{1}\right)^{2}+\left(\sigma_{2}\right)^{2}+\rho \sigma_{1} \sigma_{2}\right)$. If $W \in H_{0}^{1}(D)$, then

$$
\int_{D}(-\mathcal{L} V) W d S=\int_{D}\left((\nabla W)^{T} A \nabla V+V(b \cdot \nabla W)+c V W\right) d S
$$

We use the right-hand side of (44) for solving the parabolic problem by the finite element method. In this case, we have to impose non-homogeneous Dirichlet boundary conditions $g_{1}, g_{2}$ on the boundaries $\left(S_{1}, 0\right), S_{1} \in[0, L]$ and $\left(0, S_{2}\right)$, $S_{2} \in[0, H]$. In [31], the left-hand side of (44) is used and homogeneous Neumann boundary conditions are imposed on the boundaries $\left(S_{1}, 0\right), S_{1} \in[0, L]$ and $\left(0, S_{2}\right), S_{2} \in[0, H]$.

The functions $g_{1}, g_{2}$, used to impose Dirichlet boundary conditions, are the solutions of the one-dimensional Black-Scholes equations. For example, $g_{1}$ : $[0, L] \times[0, T] \rightarrow \mathbb{R}$ verifies

$$
\frac{\partial g_{1}}{\partial t}+\mathcal{L}_{1} g_{1} \leq 0, \quad\left(\frac{\partial g_{1}}{\partial t}+\mathcal{L}_{1} g_{1}\right)\left(\psi_{1}-g_{1}\right)=0, \quad g_{1} \geq \psi_{1}
$$

with the conditions

$$
g_{1}(0, t)=K, g_{1}(L, t)=0, g_{1}\left(S_{1}, T\right)=\psi_{1}\left(S_{1}\right)
$$

where $\psi_{1}\left(S_{1}\right)=\left(K-S_{1}\right)^{+}, \mathcal{L}_{1} g_{1}=\left(r-\delta_{1}\right) S_{1} \frac{\partial g_{1}}{\partial S_{1}}+\frac{1}{2}\left(\sigma_{1} S_{1}\right)^{2} \frac{\partial^{2} g_{1}}{\partial\left(S_{1}\right)^{2}}-r g_{1}$.

Integrating by parts, we get for all $w_{1} \in H_{0}^{1}(0, L)$

$$
\begin{aligned}
& -\int_{0}^{L}\left(\mathcal{L}_{1} g_{1}\right) w_{1} d S_{1}=\int_{0}^{L} \frac{1}{2}\left(\sigma_{1} S_{1}\right)^{2} \frac{\partial g_{1}}{\partial S_{1}} \frac{\partial w_{1}}{\partial S_{1}} d S_{1}+ \\
& +\int_{0}^{L}\left(r-\delta_{1}-\left(\sigma_{1}\right)^{2}\right) S_{1} g_{1} \frac{\partial w_{1}}{\partial S_{1}} d S_{1}+\int_{0}^{L}\left(2 r-\delta_{1}-\left(\sigma_{1}\right)^{2}\right) g_{1} w_{1} d S_{1}
\end{aligned}
$$


We use the right-hand side of the above equality for solving the parabolic problem by Algorithm 3.

The numerical parameters are: $L=H=10.5, T=5, K=4, r=0.3$, $\delta_{1}=\delta_{2}=0.1, \sigma_{1}=\sigma_{2}=0.5, \rho=0.7$.

We have applied the Algorithm 3 with a mesh of 47918 triangles and 95034 vertices, the mesh size is $h=10.5 / 200$, the time step $\Delta t=0.1$, the tolerance for the stopping test $t o l=10^{-5}$ and a fixed penalization parameter $\epsilon_{n}=10^{-3}$, for all $n \in \mathbb{N}$.

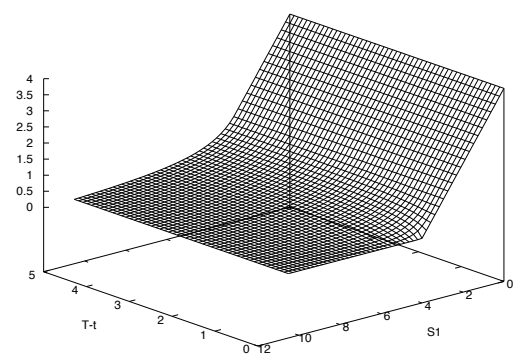

Figure 9. Test 5. The evolution of $g_{1}(\cdot, t):[0, L] \rightarrow \mathbb{R}$ from $t=T$ (foreground) to $t=0$ (background). Here, $T=5$ and $L=10.5$.

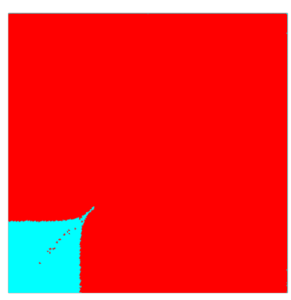

Figure 10. Test 5. Coincidence set (blue) at $t=0$.

The convergence is very fast. At the first time step the Algorithm 3 finds $g_{1}, g_{2}$ after $n=7$ iterations and the solution $V$ after $n=30$ iterations, but after $k=10$ time steps, the boundary conditions $g_{1}, g_{2}$ are computed in $n=3$ iterations and the value of the option $V$ is obtained in $n=4$ iterations.

We used a fixed mesh. Since the obstacle is non-smooth, some noise appears 


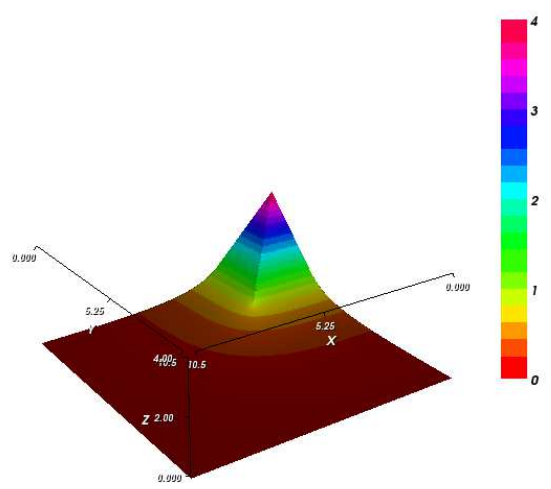

Figure 11. Test 5. The computed solution $V$ at $t=0$.

at the diagonal of Figure 10. Mesh adaptation techniques are employed as in [31] and [1].

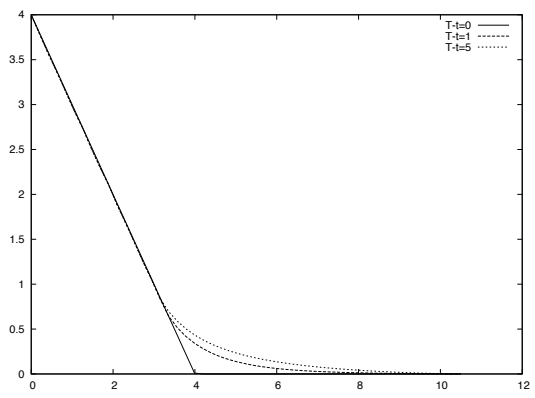

Figure 12. Test 5. The solution on the diagonal $S_{1}=S_{2}$ at $T-t=0,1,5$.

Our numerical results in this example are comparable with the augmented Lagrangian method (ALM) [11]. It is known [37] that ALM is superior to linear penalty methods or Lagrangian methods and comparable with the primal-dual active set algorithm applied to elliptic [17] or parabolic obstacle problems [18]. 


\section{Bibliography}

[1] Y. Achdou, F. Hecht and D. Pommier, A posteriori error estimates for parabolic variational inequalities, J. Sci. Comput. 37 (2008), 336-366.

[2] Y. Achdou and O. Pironneau, Computational Methods for Option Pricing, Frontiers in Applied Mathematics, 30. Society for Industrial and Applied Mathematics, Philadelphia, PA, 2005.

[3] C. Baiocchi, Su un problema di frontiera libera connesso a questioni di idraulica, Ann. Mat. Pura Appl., 92 (1972), 107-127.

[4] V. Barbu, Optimal Control of Variational Inequalities, Research Notes in Mathematics, 100. Pitman, Boston, 1984.

[5] M. Burger, N. Matevosyan and M.T. Wolfram, A level set based shape optimization method for an elliptic obstacle problem, Math. Models Methods Appl. Sci. 21 (2011), 619-649.

[6] H. Brézis, Problèmes unilatéraux, J. Math. Pures Appl. (9) 51 (1972), 1-168.

[7] H. Brézis and M. Sibony, Équivalence de deux inéquations variationnelles et applications. (French) Arch. Rational Mech. Anal. 41 (1971), 254-265.

[8] M. Broadie and J. Detemple, The valuation of American options on multiple assets, Math. Finance, 7 (1997), 241-286.

[9] G. Duvaut and J.-L. Lions, Inequalities in Mechanics and Physics, Springer-Verlag, Berlin, New York, 1976.

[10] C. M. Elliott and J. R. Ockendon, Weak and Variational Methods for Moving Boundary Problems, Research Notes in Mathematics, 59. Pitman, London, 1982.

[11] M. Fortin, R. Glowinski, Augmented Lagrangian methods. Applications to the numerical solution of boundary value problems. Studies in Mathematics and its Applications, 15. North-Holland Publishing Co., Amsterdam, 1983

[12] R. Glowinski, J.-L. Lions and R. Trémolières, Numerical Analysis of Variational Inequalities, Studies in Mathematics and its Applications, 8. North-Holland Publishing Co., Amsterdam-New York, 1981.

[13] R. Griesse and K. Kunisch, A semi-smooth Newton method for solving elliptic equations with gradient constraints, M2AN Math. Model. Numer. Anal. 43 (2009), 209238.

[14] A. Halanay C.M. Murea and D. Tiba, Existence and approximation for a steady fluid-structure interaction problem using fictitious domain approach with penalization, Mathematics and its Applications, 5 (2013), 120-147.

[15] F. Hecht, http://www.freefem.org

[16] H. Huang, W. Han and J. Zhou, The regularization method for an obstacle problem, Numer. Math. 69 (1994), 155-166. 
[17] K. Ito and K. Kunisch, Semi-smooth Newton methods for variational inequalities of the first kind, M2AN Math. Model. Numer. Anal. 37 (2003), 41-62.

[18] K. Ito and K. Kunisch, Parabolic variational inequalities: The Lagrange multiplier approach, J. Math. Pures Appl. 85 (2006), 415-449.

[19] S. L. Kamenomostskaja, On Stefan's problem. (Russian) Mat. Sb. (N.S.) 53 (95) (1961) 489-514.

[20] D. Kinderlehrer and G. Stampacchia, An Introduction to Variational Inequalities and Their Applications. Reprint of the 1980 original. Classics in Applied Mathematics, 31, SIAM, 2000.

[21] C. H. Li, A finite-element front-tracking enthalpy method for Stefan problems, IMA J. Numer. Anal. 3 (1983), 87-107.

[22] E. Lindgren, On the penalized obstacle problem in the unit half ball, Electron. J. Differential Equations, 9 (2010) 12 pp.

[23] M. Natori and H. Kawarada, An application of the integrated penalty method to free boundary problems of Laplace equation Numer. Funct. Anal. Optim. 3 (1981), 1-17.

[24] Neittaanmaki, P., Pennanen, A., Tiba, D. Fixed domain approaches in shape optimization problems with Dirichlet boundary conditions, Inverse Problems, 25 (2009), $1-18$.

[25] Neittaanmaki, P., Repin, S., Reliable methods for computer simulation. Error control and a posteriori estimates. Studies in Mathematics and its Applications, 33. Elsevier Science B.V., Amsterdam, 2004.

[26] P. Neittaanmaki, J. Sprekels and D. Tiba, Optimization of Elliptic Systems. Theory and Applications. Springer Monographs in Mathematics. Springer, New York, 2006.

[27] P. Neittaanmaki and D. Tiba, Optimal Control of Nonlinear Parabolic Systems. Theory, Algorithms, and Applications, Monographs and Textbooks in Pure and Applied Mathematics, 179. Marcel Dekker, Inc., New York, 1994.

[28] P. Neittaanmaki and D. Tiba, Fixed domain approaches in shape optimization problems, Inverse Problems, 28 (2012), 1-35.

[29] J.M. Ortega and W.C. Rheinboldt, Iterative Solution of Nonlinear Equations in Several Variables, Academic Press, New York, London, 1970.

[30] A. Petrosyan and H. Shahgholian, Parabolic Obstacle Problems Applied to Finance, in Recent developments in nonlinear partial differential equations (ed. D. Danielli), Contemp. Math., 439, Amer. Math. Soc., Providence, RI, (2007), 117-133.

[31] O. Pironneau and F. Hecht, Mesh adaption for the Black and Scholes equations, East-West J. Numer. Math. 8 (2000), 25-35.

[32] J.-F. Rodrigues, Obstacle Problems in Mathematical Physics, North-Holland Publishing Co., Amsterdam, 1987. 
[33] D. Tiba, Optimal Control of Nonsmooth Distributed Parameter Systems, Springer, Berlin, 1990.

[34] F. Wang and X.-L. Cheng, An algorithm for solving the double obstacle problems, Appl. Math. Comput. 201 (2008), 221-228.

[35] P. Wilmott, S. Howison and J. Dewynne, The Mathematics of Financial Derivatives. A Student Introduction. Cambridge University Press, Cambridge, 1995.

[36] C. S. Zhang, Adaptive Finite Element Methods for Variational Inequalities: Theory and Applications in Finance, $\mathrm{PhD}$ thesis, University of Maryland, 2007.

[37] K. Zhang, X. Q. Yang and K. L. Teo, Augmented Lagrangian method applied to American option pricing, Automatica J. IFAC 42 (2006), 1407-1416.

[38] K. Zhang, S. Wang, X. Q. Yang and K. L. Teo, A power penalty approach to numerical solutions of two-asset American options, Numer. Math. Theory Methods Appl. 2 (2009), 202-223.

\section{Author information}

Cornel Marius Murea, Laboratoire de Mathématiques, Informatique et Applications, Université de Haute Alsace, 4-6, rue des Frères Lumière, 68093 Mulhouse Cedex, France. E-mail: cornel.murea@uha.fr

Dan Tiba, Institute of Mathematics (Romanian Academy) and Academy of Romanian Scientists, Bucharest, Romania and Basque Center for Applied Mathematics, Alameda Mazarredo, 14, E-48009 Bilbao, Basque Country, Spain.

E-mail: dan.tiba@imar.ro 